\title{
PROJEÇÃO DIAMÉTRICA E VOLUMÉTRICA DA ARAUCÁRIA E ESPÉCIES ASSOCIADAS NO SUL DO PARANÁ, USANDO MATRIZ DE TRANSIÇÃO
}

\author{
Diameter and volume projection of araucaria and associated \\ species in southern Parana by using transition matrix
}

Anabel Aparecida de Mello ${ }^{1}$

Rozane de Loyola Eisfeld ${ }^{2}$

Carlos Roberto Sanquetta ${ }^{3}$

\section{Resumo}

Este estudo demonstra o desenvolvimento e a aplicação de um modelo matricial de transição em florestas de araucária, considerando dois grupos de espécies: a araucária e as folhosas. Dados para a construção do modelo foram coletados em parcelas permanentes remedidas por 3 anos em General Carneiro e Coronel Domingos Soares, Estado do Paraná. Matrizes de transição foram construídas para um horizonte temporal de 3 anos, gerando projeções para um período total de 21 anos. Aplicando-se os vetores de freqüência, os ingressos, as taxas de mortalidade e as matrizes de transição de ambos grupos, geraram-se freqüências simuladas por classe diamétrica que originaram prognoses volumétricas. As prognoses indicaram aumento de ambos os grupos na estrutura da floresta, tanto em termos de densidade como de volume. A simulação, entretanto, apontou um aumento na participação das espécies folhosas na estrutura da floresta em relação à araucária. Concluiu-se que o modelo matricial, apesar de sua simplicidade, representa bem a dinâmica da floresta de araucária. O incremento periódico anual projetado pelo modelo também foi coerente com a realidade, indicando o seu potencial preditivo.

Palavras-chave: Floresta Ombrófila Mista, Modelagem, Pinheiro, Prognose, Simulação

\section{Abstract}

This study presents the development and the application of a transition matrix to araucaria forests, taking into consideration two species groups: araucaria and broad-leaved species. To construct the model data were collected in permanent sample plots measured once again during a 3-year interval, located in General Carneiro and Coronel Domingos Soares counties, Parana State, Brazil. Transition matrices were constructed for a 3-year period, generating projections for 21 years of simulation. By applying the frequency vectors, the recruitment and mortality rates, as well as the transition matrices, it was possible to simulate projected frequencies by diameter class and volumes for both species groups. The results indicated an increase in frequency and volume of both species groups. However, the simulations showed that the participation of broad-leaved species in the forest structure would increase in a more remarkable manner as compared to araucaria. It was concluded that the matrix model (though simple) could represent adequately the dynamics of the araucaria forest. The mean periodic increment projected by the model was also coherent with reality, indicating its predictive potential. Keywords: Mixed Araucaria Forest, Modeling, Parana-pine, Stand projection, Simulation

\footnotetext{
Eng. Florestal, M.Sc., Doutoranda em Ciências Florestais, UFPR, anabel@floresta.ufpr.br

2 Eng. Florestal, Mestranda em Ciências Florestais, UFPR, rozaneloyolaeisfeld@hotmail.com

3 Eng. Florestal, M.Sc., Ph.D., Professor do Departamento de Ciências Florestais, UFPR, sanqueta@florestal.ufpr.br
} 


\section{Introdução}

A Floresta Ombrófila Mista, também conhecida como Floresta de Araucária, teve sua área de abrangência reduzida nos últimos anos em decorrência da ocupação e uso desordenado do solo no Estado do Paraná. O impulso da colonização e da atividade madeireira neste Estado deu-se após a Segunda Guerra Mundial e a expansão destas atividades tiveram como base a existência de grandes reservas de Araucaria angustifolia (SANQUETTA; TETTO, 2000).

Por este motivo, esta formação florestal, que ocupava inicialmente $73.780 \mathrm{~km}^{2}$ no Paraná, foi reduzida a $2.696 \mathrm{~km}^{2}$, em 1980 (IBDF, 1984). Estudo recente realizado por FUPEF (2000) mostra que ainda existem extensas áreas revestidas por cobertura de porte florestal no bioma da Floresta Ombrófila Mista. Essas áreas, em diferentes graus de antropização, compõem um mosaico de formações em distintas fases sucessionais e com grandes variações florísticas e estruturais. Segundo o referido trabalho, ainda existem cerca de 2,75 milhões de hectares desta formação florestal, sendo 1,28 milhão com formações pioneiras ou em estágio sucessional precoce, 1,32 milhão com formações sucessoras ou intermediárias e 150 mil hectares de florestas maturas ou próximas das florestas originais. De acordo com o estudo, que detalha mais as áreas remanescentes de Floresta Ombrófila Mista em termos de graus de antropização, cerca de $23,72 \%$ do bioma ainda é recoberto por florestas.

Um grande desafio para o Estado do Paraná é a conservação desses remanescentes de Floresta Ombrófila Mista. Ao mesmo tempo que é preciso investir no aumento da área oficialmente protegida, torna-se também necessário estimular o manejo sustentável dos recursos advindos da floresta, ou seja, um manejo ecologicamente adequado, economicamente correto e socialmente justo. Para isso, o manejo florestal deve contemplar informações biológicas, econômicas, sociais, ambientais e de mercado (SCOLFORO, 1998).

Lamentavelmente, a intensa antropização ocorrida no Paraná no último século levou atualmente à proibição do manejo de várias espécies arbóreas ditas ameaçadas de extinção pela recente Resolução emitida pelo Conselho Nacional do
Meio Ambiente ${ }^{4}$. Aquele diploma legal prescreve que serão suspensas todas as autorizações até que informações atualizadas e fidedignas sobre o real estado dos remanescentes florestais sejam aportadas. Para que o manejo florestal possa ser permitido no futuro, estudos técnico-científicos deverão ser realizados, indicando formas sustentáveis de utilizar tais recursos, sem riscos à biodiversidade.

As florestas possuem grande complexidade em termos de composição de espécies, idades e tamanhos, seja em áreas intactas, exploradas ou sujeitas a manejo. Por isto um dos importantes pontos a serem abordados é a definição do ciclo de corte e também o número de árvores por classe de diâmetro que evolui ao longo do tempo (SCOLFORO, 1998). De acordo com Sanquetta et al (1995) a projeção da estrutura diamétrica no tempo é de real importância para o manejo das florestas naturais, pois, a partir dos diâmetros futuros podem-se estimar as produções e definir as intervenções que assegurem a sustentabilidade das mesmas.

Os fatores diretamente relacionados com o desenvolvimento das distribuições diamétricas são as taxas de ingresso, crescimento e mortalidade. A projeção da estrutura diamétrica ao longo do tempo pode ser sumarizada e prognosticada eficientemente através do modelo de matriz de transição. Este modelo é um instrumento de prognose de eventos que caracteriza estados definidos de desenvolvimento que passam, a partir de um estado inicial, por uma seqüência de estados, onde a transição entre estados ocorre segundo uma certa probabilidade. Segundo Hoyos (1980), os pontos primordiais para a montagem da cadeia de Markov são: definição de estados dos sistemas e a construção da matriz de transição probabilística. O requisito para a utilização do modelo matricial é que a população possa ser dividida em estados ou compartimentos, e que haja a probabilidade de movimento de um estado para outro no tempo (ENRIGHT; OGDEN, 1979). As probabilidades que constituem os elementos da matriz de transição são determinadas pela dinâmica nas classes, ou seja, árvores de uma certa classe diamétrica que crescem para uma, duas ou mais classes consecutivas e árvores que permanecem na mesma classe ou morrem em um determinado intervalo de tempo (SANQUETTA, 1996).

${ }^{4}$ Resolução CONAMA 278/01. 
Este trabalho foi realizado com a finalidade de apresentar um modelo de projeção da distribuição diamétrica e do volume de madeira na Floresta Ombrófila Mista, tendo como estudo de caso uma área de pesquisa na região sul do Estado do Paraná. Almeja-se com o presente trabalho subsidiar a tomada de decisão a respeito do seu manejo, tendo por base a evolução temporal simulada da estrutura da floresta.

\section{Metodologia}

\section{Descrição da área de estudo}

A área de estudo compreende duas propriedades pertences às Indústrias Pedro N. Pizzatto Ltda. e ao Governo do Estado do Paraná, as quais se localizam na região sul do Paraná. A área que compreende as fazendas da empresa Pizzatto (São Pedro 1, São Pedro 2 e Santa Cândida), no município de General Carneiro, se estende entre as coordenadas $26^{\circ} 20^{\prime} 35^{\prime \prime}$ e $26^{\circ} 26^{\prime} 13^{\prime \prime}$ Latitude Sul e 51 ${ }^{\circ} 19^{\prime} 49^{\prime \prime}$ e $51^{\circ} 25^{\prime} 29^{\prime \prime}$ Longitude Oeste. As coordenadas da fazenda Lajeado Grande, também de propriedade da empresa, e do Parque Estadual das Araucárias, no município de Coronel Domingos Soares, são: $26^{\circ} 17^{\prime} 13^{\prime \prime}$ e $26^{\circ} 21^{\prime} 09^{\prime \prime}$ Latitude Sul, e $51^{\circ} 34$ '39" e $51^{\circ} 37^{\prime} 07^{\prime \prime}$ Longitude Oeste.

O clima da região é subtropical úmido mesotérmico $\mathrm{Cfb}$, segundo Koeppen, tendo temperatura média anual de $15,1^{\circ} \mathrm{C}$ e precipitação anual de cerca de $1.800 \mathrm{~mm}$. Os principais tipos de solos que ocorrem na região são Luvissolos, Cambissolos e Litossolos, de relevos suave ondulados a ondulados até montanhosos.

A formação vegetal encontrada nas áreas corresponde ao bioma denominado Floresta Ombrófila Mista ou Floresta com Araucária. Em decorrência de intervenções antrópicas, ocorrem nestas áreas condições diferenciadas da estrutura da floresta, tipos de solos e microclimas que influenciam na fisionomia da vegetação ali ocorrente.

\section{Levantamento de campo}

\section{Obtenção dos dados}

Para este estudo foram utilizadas 8 parcelas com 1,0 ha cada, quadradas (100 m x 100 m), subdivi- didas em 25 sub-parcelas de $400 \mathrm{~m}^{2}$ (20 m x $\left.20 \mathrm{~m}\right)$. As parcelas foram distribuídas de forma predeterminada, procurando abranger variações tipológicas no ambiente estudado. Dentre estas oito parcelas cinco foram instaladas nas fazendas de propriedade das Indústrias Pedro N. Pizzatto e três no Parque Estadual das Araucárias (anteriormente propriedade da empresa), no município de Coronel Domingos Soares.

No ano de implantação (1998), foram medidos os diâmetros, na forma de CAP, e alturas totais das árvores com DAP (diâmetro à altura do peito - 1,30 m) maior ou igual a $10 \mathrm{~cm}$. Procedeu-se a identificação de todas as árvores possíveis em campo e efetuou-se a coleta de material para herborização e posterior identificação em laboratório de todas as espécies encontradas nas parcelas. As árvores mortas e caídas também foram quantificadas.

As remedições destas parcelas foram feitas anualmente sempre nos meses de junho a julho dos anos subseqüentes, computando-se as árvores ingressas, ou seja, as que atingiram o DAP mínimo estipulado e as árvores mortas e caídas. Os dados foram digitados em planilha eletrônica. Para este estudo concernente à projeção da distribuição diamétrica e volumétrica foram utilizados apenas 2 anos de medições, 1998 e 2001.

\section{Modelagem}

\section{Construção da matriz de transição}

No modelo matricial de transição, para efetivar a prognose da estrutura da floresta, tem-se o volume inicial da floresta, no momento " $\mathrm{t}$ " (presente) que se conhece, e o volume a ser prognosticado num momento temporal " $\mathrm{t}+\mathrm{p}$ " (futuro). Conforme demonstrado por Sanquetta et al. (1996), para se obter a prognose volumétrica é necessário efetuar a prognose do número de árvores, a qual é dada por:

$$
\mathrm{N}_{\mathrm{t}+\mathrm{p}}=\mathrm{T}_{\mathrm{p}} \cdot \mathrm{N}_{\mathrm{t}}+\mathrm{R}_{\mathrm{p}}
$$

onde: $\mathrm{N}_{\mathrm{t}+\mathrm{p}}=$ vetor do número de árvores em classes diamétricas no ano " $\mathrm{t}+\mathrm{p}$ ";

$\mathrm{N}_{\mathrm{t}}=$ vetor do número de árvores em classes diamétricas no ano "t";

$\mathrm{T}_{\mathrm{p}}=$ matriz probabilística de transição da floresta para o período "p" ;

$\mathrm{R}_{\mathrm{p}}=$ vetor de recrutamento (ingresso) na floresta durante o período "p". 
A matriz de transição " $\mathrm{T}_{\mathrm{p}}$ ” tem a forma apresentada na Tabela 1.

Tabela 1. Estrutura da Matriz de Transição.

Table 1. Structure of the Transition Matrix

janeiro de 2000.

Table 01 - Average prices of the baia, pêra, lima oranges commercialized in the retail market of Curitiba and in its Metropolitan Region from February of 1999 to January of 2000 .

\begin{tabular}{|c|c|c|c|c|c|c|}
\hline \multirow[t]{2}{*}{ Meses } & \multicolumn{6}{|c|}{ Preço praticado $\left(\mathrm{R} \$ / \mathrm{kg}^{*}\right)$} \\
\hline & & Baia & Lima & & Pêra* & $\varepsilon * *$ \\
\hline Fevereiro & & 1,52 & 1,79 & & 0,53 & abc \\
\hline Março & & 1,04 & 0,97 & & 0,62 & $a b$ \\
\hline Abril & * & 1,24 & 0,78 & & 0,65 & a \\
\hline Maio & & 0,81 & 0,83 & & 0,40 & bc \\
\hline Junho & & 1,06 & 0,66 & & 0,45 & bc \\
\hline Julho & & 0,72 & 0,57 & & 0,43 & bc \\
\hline Agosto & & 0,83 & 0,79 & & 0,36 & $\mathrm{c}$ \\
\hline Setembro & & 1,12 & 0,90 & 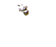 & 0,32 & $\mathrm{c}$ \\
\hline Outubro & & 1,32 & 0,78 & & 0,26 & c \\
\hline Novembro & & $* *$ & 0,78 & & 0,25 & c \\
\hline Dezembro & & 1,66 & 0,88 & & 0,27 & bc \\
\hline Janeiro & & 1,67 & 0,70 & & 0,39 & bc \\
\hline Média anual & & 1,18 & 0,87 & & 0,41 & \\
\hline
\end{tabular}

*Preços corrigidos pelo INPC-IBGE com base no mês de fevereiro de 1999.

**Cultivar não encontrada no mês.

***Médias seguidas por letras distintas diferem entre si pelo teste de Tukey ao nível de significância de $5 \%$.

Onde:

- $\mathrm{p}_{\mathrm{fi}}=\mathrm{a}$ probabilidade que uma certa árvore de uma determinada classe de diâmetro permaneça na mesma classe de diâmetro "i" durante o período "p";

- $\mathrm{p}_{\mathrm{ai}}=\mathrm{a}$ probabilidade que a planta avance para uma classe de diâmetro imediatamente maior durante o período "p" considerado;

- $\mathrm{p}_{\mathrm{bi}}=\mathrm{a}$ probabilidade que a planta avan- ce duas classes de diâmetro durante o período considerado;

- $\mathrm{n}$ = número de classes de diâmetro consideradas na matriz de transição e nos vetores de número de árvores;

- $\mathrm{m}$ =número de classes de diâmetro adicionadas até o período final da prognose.

Para se obter essas probabilidades devemse proceder aos seguintes cálculos:

$\mathrm{p}_{\mathrm{f}_{\mathrm{i}}}=\frac{\text { no.de plantas que permanecem vivas na classe de diâmetro } i \text { durante o período } p}{\text { no. de plantas da classe de diâmetro } i \text { no início do período }}$

$\mathrm{p}_{\mathrm{a}_{\mathrm{i}}}=\frac{\text { no.de plantas que avançam para a classe de diâmetro } i+1 \text { durante o período } p}{\text { no. de plantas da classe de diâmetro } i \text { no início do período }}$

no. de plantas que avançam para a classe de diâmetro $i+2$ durante o período $p$

$\mathrm{p}_{\mathrm{b}_{\mathrm{i}}}=\frac{\text { no. de plantas da classe de diâmetro } i \text { no início do período }}{\text { no }}$ 
da por:

A probabilidade de mortalidade é defini-

$$
\mathrm{p}_{\mathrm{m}_{\mathrm{i}}}=1-\mathrm{p}_{\mathrm{f}_{\mathrm{i}}}-\mathrm{p}_{\mathrm{a}_{\mathrm{i}}}-\mathrm{p}_{\mathrm{b}_{\mathrm{i}}}
$$

A aplicação das equações [1] a [6], dá origem aos vetores " $\mathrm{N}_{t+p}$ ", os quais conterão o número de árvores por classe diamétrica no futuro, isto é, as densidades prognosticadas. Neste trabalho, as simulações foram elaboradas para $3 \mathrm{em} 3$ anos e os resultados se restringem ao ano de 2022. Através das densidades prognosticadas, obtém-se a prognose de área basal, volumétrica, biomassa ou de outra variável de interesse, para os respectivos anos de simulação.

Para a construção da matriz de transição dividiu-se, inicialmente, as árvores de DAP entre 10 e $30 \mathrm{~cm}$ em dez classes diamétricas, com amplitude igual a $2 \mathrm{~cm}$; as árvores com DAP entre $30 \mathrm{e}$ $60 \mathrm{~cm}$ em 6 classes diamétricas com amplitude de $5 \mathrm{~cm}$; as árvores com DAP entre 60 e $100 \mathrm{~cm}$ em 4 classes diamétricas com amplitude de $10 \mathrm{~cm}$ e as árvores com DAP maior do que $100 \mathrm{~cm}$ em 4 classes diamétricas com amplitude de $20 \mathrm{~cm}$. As análises foram executadas separado-se as folhosas das coníferas (neste caso com apenas Araucaria angustifolia como integrante).

Para se prognosticar o volume usou-se multiplicar as seguintes variáveis:

- Área transversal: obtida a partir do diâmetro central da classe de DAP;

- Altura: obtida através de equações ajustadas por Pizatto (1999), em separado para folhosas e araucária, utilizando dados coletados na própria área;

- Volume: polinômio do $5^{\circ}$ grau ajustado para araucárias e folhosas.

\section{Programação}

\section{Desenvolvimento do simulador}

O programa utilizado para este estudo foi desenvolvido pela engenheira florestal Rozane de Loyola Eisfeld, com o objetivo de avaliar o comportamento da floresta em termos de seus processos dinâmicos e, também, para prognosticar o volume, a área basal e o número de árvores. A programação deste software foi realizada em ambiente Excelâ, utilizando o VBA (Visual Basic for Applications) como linguagem de programação. Os programas utilizados foram adquiridos legalmente pela UFPR.

\section{Resultados e discussão}

Com base nos dados das medições realizadas em 1998 e 2001, construi-se as matrizes de transição para Araucaria angustifolia e folhosas, apresentadas respectivamente nas TABELAS 1 e 2 .

Os vetores de freqüência da araucária e das folhosas, para os anos de 1998 e 2001, por sua vez, estão apresentados na TABELA 3.

Na TABELA 4 estão apresentados os valores de ingresso e mortalidade, entre os anos de 1998 e 2001, para araucária e folhosas.

A partir das matrizes de transição e dos vetores de freqüência inicial e recrutamento, foram simuladas as freqüências para os anos de 2004, 2007, 2010, 2013, 2016, 2019 e 2022, conforme mostram as TABELAS 5 e 6 . 
TABELA 1 - Matriz de transição para araucária.

TABLE 1- Transition matrix for Araucaria.

\begin{tabular}{|c|c|c|c|c|c|c|c|c|c|c|c|c|c|c|c|c|c|c|c|c|c|c|c|}
\hline \multicolumn{2}{|c|}{ Classes de DAP } & 1 & 2 & 3 & 4 & 5 & 6 & 7 & 8 & 9 & 10 & 11 & 12 & 13 & 14 & 15 & 16 & 17 & 18 & 19 & 20 & 21 & 22 \\
\hline 1 & n & 5 & 0,00 & 0,00 & 0,00 & 0,00 & 0,00 & 0,00 & 0,00 & 0,00 & 0,00 & 0,00 & 0,00 & 0,00 & 0,00 & 0,00 & 0,00 & 0,00 & 0,00 & 0,00 & 0,00 & 0,00 & 0,00 \\
\hline 2 & $12-14 \mathrm{~cm}$ & 22 & 0,66 & 0,00 & 0,00 & 0,00 & 0,00 & 0,00 & 0,00 & 0,00 & 0,00 & 0,00 & 0,00 & 0,00 & 0,00 & 0,00 & 0,00 & 0,00 & 0,00 & 0,00 & 0,00 & 0,00 & 0,00 \\
\hline 3 & $14-16 \mathrm{~cm}$ &, 02 & 33 & 63 & 00 & 0,00 & 0,00 & 0,00 & 00 & 00 & 0,00 & 0,00 & 00 & 00 & 00 & 0,00 & 0,00 & 0,00 & 0,00 & 0,00 & 00 & 00 & 00 \\
\hline 4 & 1 & $\overline{0}$ & 01 & 32 & 9 & 0,00 & 0 & 0,00 & 00 & 00 & 0,00 & 0,00 & 00 & 00 & 0,00 & 0,00 & 0,00 & 0,00 & 0,00 & 0,00 & 0,00 & 0,00 & 0,00 \\
\hline 5 & $18-20 \mathrm{~cm}$ & 00 & 0,00 & 0,05 & 0,21 & 0,69 & 0,00 & 0,00 & 0,00 & 0,00 & 0,00 & 0,00 & 0,00 & 0,00 & 0,00 & 0,00 & 0,00 & 0,00 & 0,00 & 0,00 & 0,00 & 0,00 & 0,00 \\
\hline 6 & $\mathrm{~cm}$ & 00 & 00 & 00 & 0,00 & 0,24 & 55 & 00 & 0,00 & 0,00 & 00 & 00 & 00 & 0,00 & 0,00 & 0,00 & 0,00 & 0,00 & 0,00 & 0,00 & 0,00 & 0,00 & 0,00 \\
\hline 7 & $22-24 \mathrm{~cm}$ & 0,00 & 0,00 & 0,00 & 0,00 & 0,05 & 0,33 & 0,48 & 0,00 & 0,00 & 0,00 & 0,00 & 0,00 & 0,00 & 0,00 & 0,00 & 0,00 & 0,00 & 0,00 & 0,00 & 0,00 & 0,00 & 0,00 \\
\hline 8 & $24-26 \mathrm{~cm}$ & 0,00 & 0,00 & 0,00 & 0,00 & 0,02 & 0,06 & 0,42 & 0,70 & 0,00 & 0,00 & 0,00 & 0,00 & 0,00 & 0,00 & 0,00 & 0,00 & 0,00 & 0,00 & 0,00 & 0,00 & 0,00 & 0,00 \\
\hline 9 & $26-28 \mathrm{~cm}$ & 0,00 & 0,00 & 0,00 & 0,00 & 0,00 & 0,02 & 0,03 & 0,30 & 0,56 & 0,00 & 0,00 & 0,00 & 0,00 & 0,00 & 0,00 & 0,00 & 0,00 & 0,00 & 0,00 & 0,00 & 0,00 & 0,00 \\
\hline 10 & $28-3$ & 0,00 &, 00 & 0,00 & 0,00 & 0,00 & 0,00 & 0,00 & 0,00 & 0,39 & 0,73 & 0,00 & 0,00 & 0,00 & 0,00 & 0,00 & 0,00 & 0,00 & 0,00 & 0,00 & 0,00 & 0,00 & 0,00 \\
\hline 11 & 30 & 0,00 & 0,00 & 00 & 0,00 & 0,00 & 0,00 & 0,00 & 0,00 & 0,02 & 0,27 & 0,86 & 0,00 & 0,00 & 0,00 & 0,00 & 0,00 & 0,00 & 0,00 & 0,00 & 0,00 & 0,00 & 0,00 \\
\hline 12 & $35-40 \mathrm{~cm}$ & 0,00 & 0,00 & 0,00 & 0,00 & 0,00 & 0,00 & 0,00 & 0,00 & 0,00 & 0,00 & 0,11 & 0,89 & 0,00 & 0,00 & 0,00 & 0,00 & 0,00 & 0,00 & 0,00 & 0,00 & 0,00 & 0,00 \\
\hline 13 & $40-45 \mathrm{~cm}$ & 0,00 & 0,00 & 0,00 & 0,00 & 0,00 & 0,00 & 0,00 & 0,00 & 0,00 & 0,00 & 0,01 & 0,11 & 0,86 & 0,00 & 0,00 & 0,00 & 0,00 & 0,00 & 0,00 & 0,00 & 0,00 & 0,00 \\
\hline 14 & $45-50 \mathrm{~cm}$ & 0,00 & 0,00 & 0,00 & 0,00 & 0,00 & 0,00 & 0,00 & 0,00 & 0,00 & 0,00 & 0,00 & 0,00 & 0,14 & 0,89 & 0,00 & 0,00 & 0,00 & 0,00 & 0,00 & 0,00 & 0,00 & 0,00 \\
\hline 15 & $50-$ & 0,00 & 0,00 & 00 & 0,00 & 0,00 & 0,00 & 0,00 & 0,00 & 0,00 & 0,00 & 0,00 & 0,00 & 0,00 & 0,10 & 0,83 & 0,00 & 0,00 & 0,00 & 0,00 & 0,00 & 0,00 & 0,00 \\
\hline 1 & $55-6$ & 00 & 0,00 & 0,00 & 0,00 & 0,00 & 0,00 & 00 & 00 & 0,00 & 00 & 00 & 00 & 00 & 0,00 & 0,16 & 0,86 & 0,00 & 0,00 & 0,00 & 0,00 & 0,00 & 0,00 \\
\hline 17 & $60-70 \mathrm{~cm}$ & 0,00 & 0,00 & 0,00 & 0,00 & 0,00 & 0,00 & 0,00 & 0,00 & 0,00 & 0,00 & 0,00 & 0,00 & 0,00 & 0,00 & 0,00 & 0,14 & 1,00 & 0,00 & 0,00 & 0,00 & 0,00 & 0,00 \\
\hline 18 & $70-80 \mathrm{~cm}$ & 0,00 & 0,00 & 0,00 & 0,00 & 0,00 & 0,00 & 0,00 & 0,00 & 0,00 & 0,00 & 0,00 & 0,00 & 0,00 & 0,00 & 0,00 & 0,00 & 0,00 & 0,88 & 0,00 & 0,00 & 0,00 & 0,00 \\
\hline 19 & $80-90 \mathrm{~cm}$ & 0,00 & 0,00 & 0,00 & 0,00 & 0,00 & 0,00 & 0,00 & 0,00 & 0,00 & 0,00 & 0,00 & 0,00 & 0,00 & 0,00 & 0,00 & 0,00 & 0,00 & 0,06 & 0,67 & 0,00 & 0,00 & 0,00 \\
\hline 20 & $90-100 \mathrm{~cm}$ & 0,00 & 0,00 & 0,00 & 0,00 & 0,00 & 0,00 & 0,00 & 0,00 & 0,00 & 0,00 & 0,00 & 0,00 & 0,00 & 0,00 & 0,00 & 0,00 & 0,00 & 0,00 & 0,33 & 1,00 & 0,00 & 0,00 \\
\hline 21 & $100-1$ & 0,00 & 0,00 & 00 & 0,00 & 0,00 & 0,00 & 0,00 & 0,00 & 0,00 & 0,00 & 0,00 & 0,00 & 0,00 & 0,00 & 0,00 & 0,00 & 0,00 & 0,00 & 0,00 & 0,00 & 1,00 & 0,00 \\
\hline 22 & $120-1$ & 0,00 & 0,00 & 0,00 & 0,00 & 0,00 & 0,00 & 0,00 & 0,00 & 0,00 & 0,00 & 0,00 & 0,00 & 0,00 & 0,00 & 0,00 & 0,00 & 0,00 & 0,00 & 0,00 & 0,00 & 0,00 & 1,00 \\
\hline
\end{tabular}


TABELA 2 - Matriz de transição para folhosas.

TABLE 2 - Transition matrix for broad-leaved species.

varej1sta de Curitiba e Regiao Metropolitana de tevereiro de 1999 a janeiro de 2000.

Table 02 - Average prices of the lima, tahiti and persia commercialized in the retail market of Curitiba and in its Metropolitan Region from February of 1999 to January of 2000 .

\begin{tabular}{lccc}
\hline Meses & \multicolumn{4}{c}{ Preço praticado $\left(\mathrm{R} \$ / \mathrm{kg}^{*}\right)$} \\
\cline { 2 - 5 } & Pérsia & 0,46 & $\mathrm{~d}$ \\
\hline Fevereiro & 1,70 & 0,52 & $\mathrm{~d}$ \\
Março & 1,58 & 0,52 & $\mathrm{~d}$ \\
Abril & 1,23 & 0,43 & $\mathrm{~d}$ \\
Maio & 1,54 & 0,57 & $\mathrm{~d}$ \\
Junho & 1,13 & 0,53 & $\mathrm{~d}$ \\
Julho & 1,27 & 1,13 & $\mathrm{bc}$ \\
Agosto & 0,74 & 1,32 & $\mathrm{ab}$ \\
Setembro & 1,18 & 0,92 & $\mathrm{c}$ \\
Outubro & 1,04 & 1,48 & $\mathrm{a}$ \\
Novembro & $* *$ & 0,98 & $\mathrm{bc}$ \\
Dezembro & 1,80 & 0,47 & $\mathrm{~d}$ \\
Janeiro & 1,82 & 0,78 & \\
\hline Média anual & 1,37 & \multicolumn{3}{c}{0}
\end{tabular}

*Preços corrigidos pelo INPC-IBGE com base no mês de feverëiro de 1999.

**Cultivar não encontrada no mês.

***Médias seguidas por letras distintas diferem entre si pelo teste de Tukey ao nível de significância de $5 \%$. 
TABELA 3 - Vetores de freqüências observadas para os anos de 1998 e 2001.

TABLE 3-Vectors of observed frequencies for the years of 1998 and 2001.

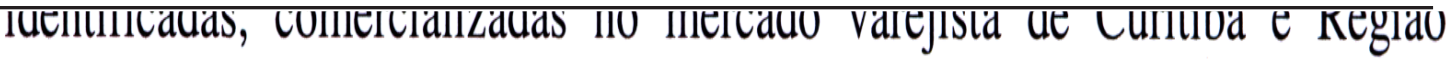
Metropolitana de fevereiro de 1999 a janeiro de 2000.

Table 03 - Average prices of the tangerine ponkan, murcote and other cultivates including those not identified commercialized in the retail market of Curitiba and in its Metropolitan Region from February of 1999 to January of 2000.

\begin{tabular}{|c|c|c|c|c|}
\hline \multirow[t]{2}{*}{ Meses } & \multicolumn{4}{|c|}{ Preço praticado $\left(\mathrm{R} \$ / \mathrm{kg}^{*}\right)$} \\
\hline & Murcote*** & Ponkan & $\begin{array}{c}\text { Outras } \\
\text { cultivares }\end{array}$ & $\begin{array}{l}\text { Cultivares não } \\
\text { identificadas }\end{array}$ \\
\hline Fevereiro & $2,10 a$ & $* *$ & $* *$ & $* *$ \\
\hline Março & $1,91 \mathrm{ab}$ & $1,18 \mathrm{a}$ & 0,83 & 2,66 \\
\hline Abril & $1,83 a b c$ & $1,05 \mathrm{a}$ & 0,76 & 3,04 \\
\hline Maio & $1,67 a b c$ & $0,28 \mathrm{~b}$ & 0,65 & 0,71 \\
\hline Junho & $1,60 a b c$ & $0,23 \mathrm{~b}$ & 0,41 & 0,76 \\
\hline Julho & $0,46 \quad c$ & $0,28 \mathrm{~b}$ & 0,69 & 0,96 \\
\hline Agosto & $0,54 \quad c$ & $0,32 \mathrm{~b}$ & 0,92 & 0,47 \\
\hline Setembro & $0,55 \quad c$ & $* *$ & 0,83 & 0,86 \\
\hline Outubro & $0,57 \quad c$ & $* *$ & 0,93 & 0,85 \\
\hline Novembro & $0,85 \quad b c$ & $* *$ & $* *$ & $* *$ \\
\hline Dezembro & $0,94 \quad b c$ & $* *$ & $* *$ & $* *$ \\
\hline Janeiro & $* *$ & $* *$ & $* *$ & $* *$ \\
\hline Média anual & 1,19 & 0,56 & 0,75 & 1,29 \\
\hline
\end{tabular}

*Preços corrigidos pelo INPC-IBGE com base no mês de fevereiro de 1999.

**Cultivar não encontrada no mês. 
TABELA 4 - Ingresso e mortalidade no período de estudo.

TABLE 4 - Recruitment and mortality in the study period.

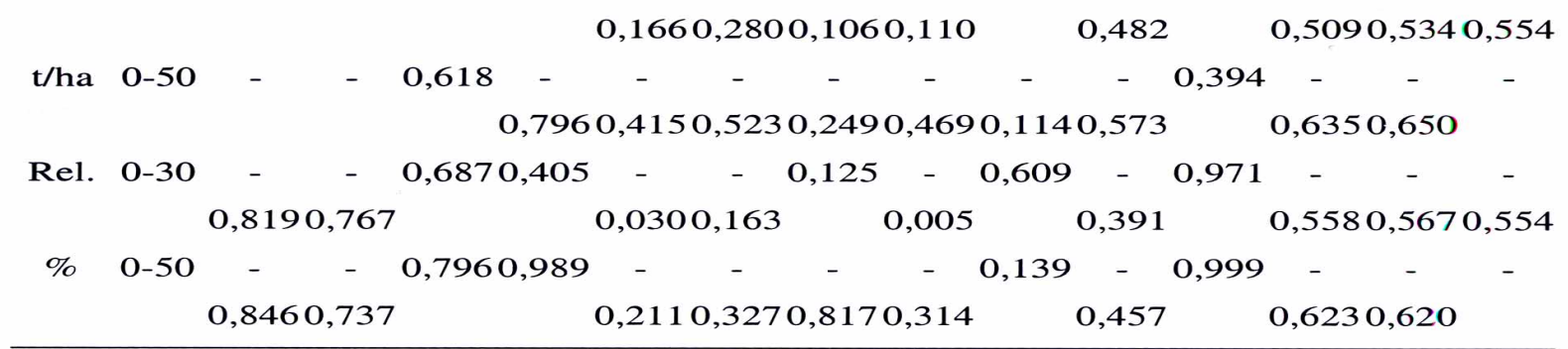

Onde: Val. = Valores; Abs. = absolutos; Rel. = relativos; Prof. = profundidade $\mathrm{em} \mathrm{cm}$.

TABELA 5 - Freqüências simuladas para os anos de 2004 a 2022 para araucária.

TABLE 5-Simulated frequencies for araucaria for the years 2004 to 2022.

\begin{tabular}{|c|c|c|c|c|c|c|c|c|c|}
\hline \multicolumn{2}{|c|}{ Classes de DAP } & 2001 & 2004 & 2007 & 2010 & 2013 & 2016 & 2019 & 2022 \\
\hline 1 & $10-12 \mathrm{~cm}$ & 71 & 86 & 97 & 105 & 111 & 116 & 119 & 122 \\
\hline 2 & $12-14 \mathrm{~cm}$ & 57 & 53 & 53 & 56 & 59 & 63 & 66 & 69 \\
\hline 3 & $14-16 \mathrm{~cm}$ & 64 & 61 & 58 & 56 & 56 & 57 & 59 & 62 \\
\hline 4 & $16-18 \mathrm{~cm}$ & 67 & 74 & 79 & 82 & 83 & 85 & 86 & 88 \\
\hline 5 & $18-20 \mathrm{~cm}$ & 53 & 54 & 55 & 57 & 59 & 61 & 62 & 64 \\
\hline 6 & $20-22 \mathrm{~cm}$ & 40 & 35 & 32 & 31 & 30 & 31 & 31 & 32 \\
\hline 7 & $22-24 \mathrm{~cm}$ & 34 & 32 & 30 & 28 & 27 & 26 & 26 & 26 \\
\hline 8 & $24-26 \mathrm{~cm}$ & 36 & 43 & 47 & 49 & 49 & 48 & 48 & 48 \\
\hline 9 & $26-28 \mathrm{~cm}$ & 33 & 31 & 32 & 33 & 35 & 35 & 36 & 36 \\
\hline 10 & $28-30 \mathrm{~cm}$ & 43 & 44 & 44 & 45 & 46 & 47 & 48 & 49 \\
\hline 11 & $30-35 \mathrm{~cm}$ & 72 & 74 & 77 & 79 & 80 & 82 & 84 & 86 \\
\hline 12 & $35-40 \mathrm{~cm}$ & 92 & 90 & 89 & 88 & 88 & 87 & 87 & 88 \\
\hline 13 & $40-45 \mathrm{~cm}$ & 78 & 78 & 77 & 77 & 77 & 76 & 76 & 76 \\
\hline 14 & $45-50 \mathrm{~cm}$ & 67 & 71 & 74 & 76 & 79 & 81 & 83 & 84 \\
\hline 15 & $50-55 \mathrm{~cm}$ & 54 & 51 & 49 & 48 & 47 & 46 & 46 & 46 \\
\hline 16 & $55-60 \mathrm{~cm}$ & 45 & 47 & 48 & 49 & 49 & 49 & 50 & 50 \\
\hline 17 & $60-70 \mathrm{~cm}$ & 47 & 53 & 60 & 67 & 74 & 81 & 88 & 95 \\
\hline 18 & $70-80 \mathrm{~cm}$ & 15 & 13 & 12 & 10 & 9 & 8 & 7 & 6 \\
\hline 19 & $80-90 \mathrm{~cm}$ & 3 & 3 & 3 & 2 & 2 & 2 & 2 & 2 \\
\hline 20 & $90-100 \mathrm{~cm}$ & 2 & 3 & 4 & 5 & 6 & 6 & 7 & 8 \\
\hline 21 & $100-120 \mathrm{~cm}$ & 1 & 1 & 1 & 1 & 1 & 1 & 1 & 1 \\
\hline 22 & $120-140 \mathrm{~cm}$ & 3 & 3 & 3 & 3 & 3 & 3 & 3 & 3 \\
\hline
\end{tabular}


Na TABELA 5, percebe-se o progressivo aumento do número de árvores nas menores classes de DAP, de acordo com a simulação pelo modelo matricial. Este aumento é atribuído ao recrutamento ser maior que a mortalidade para a espécie. Percebe-se também na tabela as evoluções nas freqüências simuladas com o passar do tempo, determinando expressivos aumentos em algumas classes, com especial destaque à classe 17 (60-70 $\mathrm{cm}$ ), o que denota o padrão de crescimento superior à mortalidade que a espécie apresentaria para o horizonte temporal da simulação.

TABELA 6 - Freqüências simuladas para os anos de 2003 a 2013 para folhosas.

TABLE 6 -Simulated frequencies for broad-leaved species for the years 2003 to 2013.

TABLE 6 - Simulated frequencies for broad-leaved species for the years from 2003 to 2013

\begin{tabular}{|c|c|c|c|c|c|c|c|c|c|}
\hline \multicolumn{2}{|c|}{ Classes de DAP } & 2001 & 2004 & 2007 & 2010 & 2013 & 2016 & 2019 & 2022 \\
\hline 1 & $10-12 \mathrm{~cm}$ & 580 & 702 & 792 & 859 & 908 & 944 & 970 & 990 \\
\hline 2 & $12-14 \mathrm{~cm}$ & 338 & 344 & 373 & 410 & 449 & 484 & 516 & 542 \\
\hline 3 & 14-16cm & 366 & 361 & 362 & 372 & 390 & 414 & 441 & 468 \\
\hline 4 & $16-18 \mathrm{~cm}$ & 263 & 256 & 251 & 248 & 249 & 254 & 262 & 274 \\
\hline 5 & $18-20 \mathrm{~cm}$ & 223 & 234 & 240 & 243 & 245 & 247 & 250 & 256 \\
\hline 6 & $20-22 \mathrm{~cm}$ & 170 & 174 & 179 & 184 & 188 & 191 & 194 & 197 \\
\hline 7 & $22-24 \mathrm{~cm}$ & 119 & 123 & 127 & 131 & 134 & 138 & 141 & 143 \\
\hline 8 & $24-26 \mathrm{~cm}$ & 96 & 98 & 101 & 103 & 106 & 109 & 112 & 115 \\
\hline 9 & $26-28 \mathrm{~cm}$ & 72 & 78 & 83 & 87 & 91 & 94 & 97 & 100 \\
\hline 10 & $28-30 \mathrm{~cm}$ & $65 *$ & 72 & 78 & 84 & 90 & 95 & 99 & 103 \\
\hline 11 & $30-35 \mathrm{~cm}$ & 69 & 70 & 73 & 78 & 82 & 88 & 93 & 98 \\
\hline 12 & $35-40 \mathrm{~cm}$ & 43 & 48 & 51 & 55 & 59 & 62 & 66 & 71 \\
\hline 13 & $40-45 \mathrm{~cm}$ & 32 & 36 & 40 & 44 & 47 & 51 & 56 & 60 \\
\hline 14 & $45-50 \mathrm{~cm}$ & 20 & 23 & 26 & 30 & 34 & 38 & 42 & 46 \\
\hline 15 & $50-55 \mathrm{~cm}$ & 15 & 16 & 18 & 20 & 23 & 25 & 28 & 32 \\
\hline 16 & $55-60 \mathrm{~cm}$ & 11 & 12 & 13 & 14 & 16 & 18 & 19 & 21 \\
\hline 17 & $60-70 \mathrm{~cm}$ & 16 & 14 & 13 & 11 & 10 & 9 & 8 & 7 \\
\hline 18 & $70-80 \mathrm{~cm}$ & 7 & 8 & 8 & 8 & 8 & 8 & 7 & 7 \\
\hline 19 & $80-90 \mathrm{~cm}$ & 5 & 6 & 7 & 9 & 10 & 11 & 13 & 14 \\
\hline 20 & $90-100 \mathrm{~cm}$ & 5 & 5 & 5 & 5 & 5 & 5 & 5 & 5 \\
\hline 21 & $100-120 \mathrm{~cm}$ & 1 & 1 & 1 & 1 & 1 & 1 & 1 & 1 \\
\hline 22 & $120-140 \mathrm{~cm}$ & 1 & 1 & 1 & 1 & 1 & 1 & 1 & 1 \\
\hline 23 & $140-160 \mathrm{~cm}$ & 0 & 0 & 0 & 0 & 0 & 0 & 0 & 0 \\
\hline 24 & $160-180 \mathrm{~cm}$ & 0 & 0 & 0 & 0 & 0 & 0 & 0 & 0 \\
\hline 25 & $>180 \mathrm{~cm}$ & 1 & 1 & 1 & 1 & 1 & 1 & 1 & 1 \\
\hline
\end{tabular}

Para as espécies folhosas (TABELA 6) percebe-se um progressivo aumento do número de árvores em todas as classes de DAP, decorrente da simulação com o modelo matricial, o que ocorre devido ao recrutamento elevado e ao bom crescimento que estas espécies apresentariam no horizonte de simulação.
Nas TABELAS 5 e 6 pode-se verificar que as folhosas apresentam a distribuição decrescente (tipo "J" invertido), ou seja, um número de árvores bem maior nas classes de diâmetro menores. Este padrão de distribuição é esperado para florestas naturais onde o processo contínuo de regeneração e competição no sub-bosque se verifica de 
forma balanceada. Já para araucária, verifica-se uma distribuição distinta, com tendência uniforme em termos de freqüência nas classes diamétricas, indicando que, embora o recrutamento da espécie venha sendo maior que a respectiva mortalidade, em longo prazo a estabilidade da população é menor, determinando ameaças maiores à esta espécie em relação às espécies folhosas.
Comparando ambas tabelas é possível evidenciar a tendência de um desempenho arrojado das folhosas em termos de evolução na estrutura da floresta em comparação à araucária. Tal situação é também ressaltada na FIGURA 1, que mostra as prognoses futuras para a variável de volume total de madeira de fuste com casca por hectare.

FIGURA 1 - Prognose volumétrica para araucária e folhosas

FIGURE 1 - Volumetric Prognosis for Arancaria and broad-leaved species

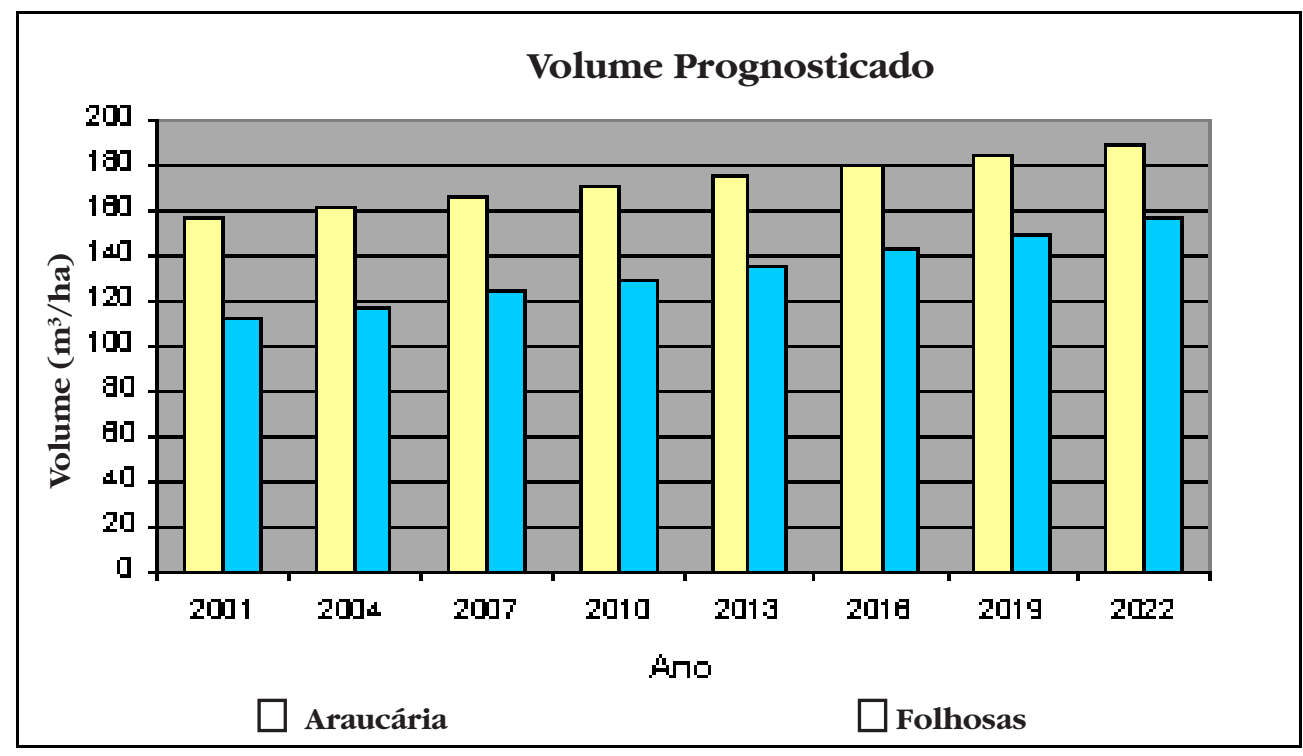

Conforme observado na FIGURA 1, o modelo matricial projeta um acréscimo do volume de madeira de araucária em 21 anos, passando de cerca de $157 \mathrm{~m}^{3} /$ ha em 2001 a cerca de $189 \mathrm{~m}^{3} /$ ha em 2022. Para folhosas, o modelo também estimou um acréscimo volumétrico ainda mais marcante, de cerca de $112 \mathrm{~m}^{3} /$ ha em 2001 para cerca de $156 \mathrm{~m}^{3} /$ ha em 2022. No cômputo de ambos, a floresta apresentou um crescimento de $269 \mathrm{~m}^{3} / \mathrm{ha}$ para $346 \mathrm{~m}^{3} / \mathrm{ha}$, resultando em um incremento de $3,67 \mathrm{~m}^{3} /$ ha.ano ${ }^{-1}$, o qual é usualmente encontrado em florestas mistas de araucária em condições semelhantes às reportadas no presente estudo.

O presente estudo se fundamenta apenas em uma única área de estudo e em horizonte temporal restrito. Por isso, discussões mais amplas são arriscadas e impertinentes. Todavia, algumas considerações julgadas relevantes podem ser levanta- das. As prognoses apontam para uma maior participação das folhosas na estrutura da floresta com o passar do tempo em função das componentes de dinâmica florestal (ingresso, crescimento e mortalidade) serem menos favoráveis à araucária. Sanquetta e Mattei (2002) e Sanquetta (2000) debatem o menor poder competitivo da araucária em relação a outras espécies, a pequena regeneração natural que a espécie apresenta em condições normais e a perda de vitalidade dos indivíduos adultos sem a conseqüente reposição dos estoques que garantam a hegemonia da espécie hoje predominante na comunidade. Estudos recentes que abordam a dinâmica de florestas de araucária (Barth Filho, 2002; Pizatto, 1999) também tratam da questão da regeneração da espécie, mostrando situações diferenciadas em florestas semelhantes, mas em locais distintos. Será pre- 
ciso refinar o presente modelo, alimentando-o com mais dados, para poder avaliar se a tendência aqui mostrada é regra ou não.

Como dito previamente, generalizações e comentários mais contundentes não seriam plausíveis, mas pode-se dizer que a tendência apresentada pelo modelo matricial em questão parece ser coerente com a realidade. É ainda digno de menção que o incremento periódico anual prognosticado pelo modelo matricial é compatível com valores observados na prática (SANQUETTA, 1998).

\section{Conclusões}

Através dos resultados apresentados neste trabalho pode-se concluir que:

- O modelo matricial de transição representa de forma coerente o processo de dinâmica na floresta de araucária;

- As prognoses com o modelo matricial indicam uma evolução positiva de ambos grupos (araucária e folhosas) em termos de densidade e volume, havendo uma tendência de as folhosas aumentarem sua participação na estrutura da floresta;

- O incremento periódico anual prognosticado pelo modelo matricial é comparável a valores observados em outros estudos, sendo um bom indicativo de seu desempenho e poder preditivo.

\section{Referências}

BARTH FILHO, N. Monitoramento do crescimento e da produção em Floresta Ombrófila Mista, utilizando parcelas permanentes. $87 \mathrm{f}$. Dissertação (Mestrado em Ciências Florestais) Setor de Ciências Agrárias, Universidade Federal do Paraná. Curitiba, 2002.
ENRIGHT, N.; OGDEN, J. Applications of transition matrix models in forest dynamics: Araucaria in Papua New Guinea and Nothofagus in New Zealand. Australian Journal of Ecology, n. 4, p. 3-23. 1979.

HOYOS, A. 1980. Processos estocásticos e previsão. In: SIMPÓSIO NACIONAL DE PROBABILIDADE E ESTATÍSTICA, 4., Anais... Rio de Janeiro, 1980.

IBDF - INSTITUTO BRASILEIRO DE DESENVOLVIMENTO FLORESTAL. Inventário Nacional. Florestas Nativas-Paraná e Santa Catarina. Brasília, 1984. 345 p.

PIZATTO, W. Avaliação biométrica da estrutura e da dinâmica de uma Floresta Ombrófila Mista em São João do Triunfo - PR: 1995 a 1998. 170 f. Dissertação (Mestrado em Ciências Florestais) Setor de Ciências Agrárias, Universidade Federal do Paraná. Curitiba, 1999.

SANQUETTA, C.R.; ANGELO, H.; BRENA, D.A.;M ENDES, J.B. Predição da distribuição diamétrica, mortalidade e recrutamento de floresta natural com matriz markoviana de potência. Floresta. Curitiba, v. 1/2, n. 24, p. 23-26, 1995.

SANQUETTA, C.R. Fundamentos biométricos dos modelos de simulação florestal. FUPEF Série Didática n. 8. Curitiba, 1996. 49 p.

Inventário contínuo em floresta

natural: implantação de um sistema de monitoramento de crescimento e produção - Relatório de Pesquisa. Curitiba: FUPEF, 1998.

SANQUETTA, C.R. ARAUSIS: sistema de simulação para manejo sustentável de florestas de araucária. Floresta, Curitiba, v. 29, n. 1, p. 115 - 121, 2000.

SANQUETTA, C.R.; TETTO, A.F. Pinheiro-doParaná: lendas e realidades. Curitiba, 2000. 112 p.

SANQUETTA, C.R.; MATTEI, E. Manejo racional da Floresta de Araucária. Revista Meio Ambiente Santa Catarina, Florianópolis, v. 2, p. 58, 2002.

SCOLFORO, J.R.S. Manejo Florestal. Lavras: FAEPE, 1998. $438 \mathrm{p}$. 